\title{
Robot-assisted kidney transplantation: a single-center experience
}

\author{
Seoungjun Lim, Youngmin Ko, Hyeeun Kwon, Joohee Jung, Hyunwook Kwon, Younghoon Kim, Sung Shin
}

Department of Surgery-Transplantation, Asan Medical Center, University of Ulsan College of Medicine, Seoul, Korea

Background: Although the open approach is the standard procedure for kidney transplantation, robotic-assisted kidney transplantation (RAKT) has been recently adopted and are becoming popular, especially in Western countries. Different from previous reports, we have developed surgical procedures for RAKT without flipping. The aim of this study is to introduce the step-by-step procedures for RAKT without flipping and to evaluate surgical outcomes of RAKT at our center.

Methods: This was a single-center retrospective study of 38 patients who underwent RAKT from a living donor between August 2020 and July 2021. Dissection of the external iliac vessels was performed by conventional transperitoneal approach. However, to avoid graft flapping to retroperitoneal pouch, we initially placed graft lateral to the iliac vessels. Venous/arterial anastomosis and ureterovesical anastomosis was performed by totally intracorporeal. There was no delayed graft function.

Results: A total of 38 patients had RAKT at our center during the study period. The mean age and body mass index of recipients were 43.2 years (16-68 years) and $23.7 \mathrm{~kg} / \mathrm{m}^{2}\left(16.0-41.2 \mathrm{~kg} / \mathrm{m}^{2}\right)$, respectively. Thirteen cases $(31.7 \%)$ of ABO incompatible and four cases $(9.8 \%)$ of T or B flow cytometry-positive RAKT were performed. There was one case of primary non-function due to renal vein thrombosis and one case of mortality due to respiratory arrest which was not related to surgical procedures. The mean vascular anastomosis time and rewarming time were 50.7 minutes (36-69 minutes) and 65.7 minutes (42-119 minutes), respectively. Operative time was 308.1 minutes (196-422 minutes). A patient was hospitalized for an average of 7.1 days after RAKT. The mean estimated glomerular filtration rate one month after RAKT was $72.8 \mathrm{~mL} / \mathrm{min} / \mathrm{m}^{2}$.

Conclusions: Considering the lower risk of surgical complications, favorable cosmetic aspects, and earlier recovery, as well as comparable clinical outcomes with conventional open techniques, RAKT is a feasible option for those with end-stage renal disease.

Corresponding author: Sung Shin

E-mail: basest@naver.com

(C) The Korean Society for Transplantation

This is an Open Access article distributed under the terms of the Creative Commons Attribution Non-Commercial License (http://creativecommons.org/licenses/by-nc/4.0/) which permits unrestricted non-commercial use, distribution, and reproduction in any medium, provided the original work is properly cited. 